\title{
Human endogenous retroviruses: Their possible role in the molecular etiology of the schizophrenia*
}

\author{
Raúl Alelú-Paz ${ }^{1 \#}$, Ignacio Iturrieta-Zuazo ${ }^{2 \#}$ \\ ${ }^{1}$ Department of Biochemistry and Molecular Biology, School of Medicine, University of Alcalá, Madrid, Spain \\ ${ }^{2}$ Department of Anatomy and Embriology, School of Medicine, San Pablo CEU University, Madrid, Spain \\ Email: raul.alelu@uah.es
}

Received 9 November 2011; revised 11 December 2011; accepted 20 January 2012

\begin{abstract}
Human endogenous retroviruses (HERVs) represent footprints of previous retroviral infections. They are integrated within the human germ line and constitute approximately $8 \%$ of our genome. They have the potential to harm, given their capacity to alter the cellular metabolism, and could be involved in various pathological processes. This revision intends to highlight the importance of HERVs in health and disease, and the increasing interest of the scientific community in their biology. In this overview, we will present a brief summary of the structure and physiological function of HERVs and an analysis of their role in schizophrenia, a paradigm of mental illness, particularly stressing the importance of HERV research to explore the more basic mechanisms disrupted in this psychiatric condition.
\end{abstract}

Keywords: HERVs; Schizophrenia; Molecular Biology

\section{INTRODUCTION TO ENDOGENOUS HUMAN RETROVIRUSES (HERVs)}

Retroviruses are enveloped viruses that contain two single stranded ribonucleic acid (RNA) genomes. The virus uses its own reverse transcriptase (RT) to convert its RNA into linear double-stranded deoxyribonucleic acid (DNA), which can then be integrated into the chromosomal DNA of the host cell and expressed. Retroviruses maintain their genetic material in two ways, either as viral RNA in viral particles or as a provirus integrated into its host's DNA [1,2].

It is well know that retroviruses can not only exist as exogenous pathogens but also as endogenous retroviruses, and those that are integrated within the human genome are called human endogenous retroviruses (HERVs). These

*Competing interests: The authors declare that they have no competing interests.

"Both authors contributed equally to this work. viruses were discovered during the eighties $[3,4]$ and it is commonly accepted that HERVs represent footprints of previous retroviral infection. The accumulated infection by different exogenous retroviruses (and their reinfection) and the intracellular amplification of endogenous retroviral sequences, results in about $8 \%$ of the human genome being of retroviral origin $[5,6]$.

Exogenous retroviruses usually infect somatic cells and they cannot be transmitted to the host offspring. However, on rare occasions, when the retroviruses overcome the numerous restricting factors, germ cells can be infected by exogenous retroviruses that integrate their genetic material into the chromosomes of the germ cells. Thus, if the germ cell survives, the integrated retroviruses can be transmitted and inherited in a mendelian fashion, vertically through the germ line over successive generations [7]. The process of stable integration into a host genome's is called endogenization and this process is limited by several factors, such as the hosts immune system [8], innate antiretroviral protein activity, e.g. TRIM $5 \alpha$ [9], APOBEC3G [10], miRNA [11], the presence of an adequate viral receptor expressed in the germ line that permits the entrance of the virus, etc. [12].

After endogenization, most HERVs are not infectious as they are usually inactivated by stop codons, frame shift mutations or deletions [13]. However, despite the apparent lack of infectious activity, HERVs have been very important in the evolution and divergence of hominids, as they may contribute to genomic plasticity [14].

\subsection{HERV Structure}

The genomic organization of integrated HERVs is similar to that of exogenous retroviruses, with two identical long terminal repeats (LTRs) flanking the coding sequences. LTRs are mainly responsible for the regulatory potential of HERVs since they contain a promoter, enhancer and the repressor sequences for tissue-specific expression, as well as the polyadenylation signal [7]. The LTR activity essentially depends on the chromatin and 
CpG methylation status of the promoter and regulatory regions [15]. Moreover, LTRs contain many regulatory sequences such as transcription factor binding sites, e.g., for HRE, YYI, CBF/NF1, TBF, C/EBP, etc. [16].

Full length HERVs encode 3 main genes, pol, gag and $e n v$, as well as the previously cited LTRs. These genes are processed and expressed as polyproteins. The gag (group specific antigen) gene encodes structural capsid proteins while pol encodes the protease, the polymerase and the reverse transcriptase that facilitates the synthesis of DNA complementary to the viral RNA. Finally, the envelope protein, env, is needed for viral budding and infection [7].

\subsection{Physiological Function}

Although we are still far from fully understanding the physiological activity of HERVs and how they are regulated, the information about HERVs is increasing constantly. Indeed, it has been proposed that a HERV transcriptome project will be necessary to comprehend the transcriptional activity of HERVs [15]. Different studies have indicated that HERV transcription can be modulated and activated by a number of environmental and physiological factors, such as UV light irradiation [17], cell infection [18], steroid hormones [19], transcription factors, cytokines [20], and also by genotoxic and cytotoxic xenobiotics such as 5-azacytidine [21], etc.

There is evidence that many HERV promoter sequences still display transcriptional activity after millions of years in the genome. In fact, it was recently demonstrated that there is virtually no human tissue that lacks HERV transcripts, and transcripts from several HERV families are usually found in all human tissue [15]. Under normal conditions, the physiological activity of cells may include the transcription and transduction of HERVs. Moreover, their pattern of expression depends on the type of cell and tissue, as well as on the developmental stage, and hence, they are subjected to very precise temporal and spatial regulation [22]. Indeed, cell type-dependent expression was found to be due to specific regulatory sequences principally located in the U3 region of the LTRs [23].

Since HERVs have been present in our genome for a very long period of time, they constitute part of our molecular evolution [16]. Indeed, HERVs are thought to have played an important role in the evolution of mammalian genomes, such that their presence may be beneficial to the human host. In this respect, there are several examples of their participation in important and complex biological processes, such as placental development and embryogenesis [24]. On the other hand, like any other genomic element, aberrant activity of HERvs could have fatal consequences, leading to the appearance of different pathologies [25].

\subsection{Pathological Mechanisms}

Although it remains controversial as to whether HERVs are markers or triggers of any human disease, there is no doubt that HERVs have the potential to harm, given their capacity to alter the cellular functions. In this respect several mechanisms by which HERVs could produce pathological effects have been proposed, some of which are summarized below:

1) Generation of new variants of HERVs. Although most HERVs cannot be transmitted horizontally, it is possible that pathogenic variants can be generated by recombination with other endogenous or exogenous sequences. For example, the exchange of envelope protein sequences could generate variants that mediate infection via a different receptor, expanding the host range [25]. In fact, it has already been possible to generate a replication competent HERV-K [26].

2) Insertional mutagenesis. Since HERVs may potentially undergo retrotransposition, gene activity could be altered if the HERV integrates into or near to a coding or regulatory sequence. Such retrotransposition may occur either through a retroviral reverse transcriptase (RT) or the RT encoded by Long Interspersed Nuclear Element (LINE), a non-LTR retrotransposon family [27]. If HERVencoded components associate with, or otherwise modify, cellular proteins or structures, it is possible to produce new types of molecules that in some cases could be autoantigenic [28]. Thus, these insertions have the potential to generate neo-antigens or candidate tumor- associated antigens similar to HERV-H Xp22.3 ORF or HERV$\mathrm{K}$ env, which has been described as trigger of specific immune responses in gastrointerstinal and breast cancer patients [29]. In this respect, HERVs could stimulate the immune response because they are capable of producing epitope spread and activation of innate immunity through pattern recognition receptors, this being related with proinflammatory cytokine secretion [28]. They could also generate active splice sites and open reading frames that could lead to aberrant splice variants and fusion proteins with viral sequences [25]. It is also possible that HERVs insertional activity may cause the appearance of superantigens. Indeed, it has been proposed that some HERVs could participate in the generation of molecules that bypass the normal route for the major histocompatibility complex (MHC)-peptide-T cell receptor interaction in the binding of a given $\mathrm{T}$ cell $\mathrm{V} \beta$ chain and MHC II protein [20]. Insertional mutagenesis also contemplates the effects of LTRs as cis-regulatory elements. Solitary LTRs are potential modulators and their activity as a transcriptional control region might extend to other genes, fulfillling the function of a promoter or enhancer, or promoting 
polyadenylation, possibly in a tissue and development stage specific manner [22]. Such LTR enhancer activity could alter the transcriptional profiles of the pre-existing neighboring genes and their polyadenylation sites may cause abnormal termination of the read-through transcript [30].

3) Protein toxicity. Accessory protein expression may affect cellular metabolism. HERVs could also encode retroviral proteins used by exogenous retroviruses, thereby expanding their infection [31] and possibly causing cell damage. Molecular mimicry could also cause cell damage, and the expression of retroviral proteins that crossreact with components of the MHC could disrupt the idiotypic network that regulates the immune system, resulting in autoimmunity [28].

These mechanisms may play a role in certain types of cancer and autoimmune diseases, such as lupus erythematosus, insulin-dependent diabetes mellitus, multiple sclerosis, Sjögren's syndrome, and rheumatoid arthritis, among others [32]. With respect to severe mental illnesses, modern psychiatric diagnosis systems are based on the neo-Kraepelinian assumptions that have created explicit diagnosis criteria for different clinical manifestations. These disorders are currently considered as unitary cli- nical entities in Sydenham's terms, although it has not described a proper anatomopathology. The data obtained in the different research fields are, in the great majority of cases, debatable, determining that the huge mass of evidence obtained is not perceived as an indication of a continuing increase of knowledge, but it is seen as a sign of uncertainty and confusion, so we think that it is important to explore new research proposals, such as the role of HERVs in the pathophysiology of schizophrenia.

\section{HUMAN ENDOGENOUS RETROVIRUSES AND SCHIZOPHRENIA}

Adoption, family and twin studies have demonstrated the contribution of genetic factors to the risk of developing schizophrenia, a disease with a strong and complex genetic component, evident through the increased incidence of schizophrenia in relatives, fraternal and identical twins of patients. Likewise, a number of environmental factors have been proposed to increase the risk for schizophrenia, such as maternal influenza during the second trimester of pregnancy [33].

Although tremendous progress has been achieved in this field, the main molecular determinants of the disease remain unclear. Accordingly, more recent attention has focused on the study of the role of HERVs in the pathogenesis of schizophrenia [34,35], particularly centered on two particular HERV families, HERV-W and HERV-K.

\subsection{HERV-W and Schizophrenia}

HERV-W exists in multiple copies in the human genome [36]. Although the majority of the HERV-W gag, pol and env genes have lost their ability to encode viral proteins, an element on chromosome 7q21 has been described that contains an intact env gene. This gene encodes an envelope protein that has been ascribed a role in placental biogenesis and the aberrant expression of this protein is associated with preeclampsia and multiple sclerosis [37]. Likewise, expression of this HERV gene was recently described in several brain areas, such as the cerebral cortex, brain stem or thalamus [38]. Moreover, the HERV-W family has been implicated in schizophrenia following the analysis of this element in the pathogenesis of the disease in post mortem material, blood samples and cerebrospinal fluid (CSF) (Table 1) [35,37, 39-43].

In this respect, abnormal expression of HERV genes has been found in samples from patients diagnosed with schizophrenia (both at first onset disorder and in later stages of the disease), although there are some contradictory aspects of such reports. In the majority of cases described, the expression of HERV-W was abnormal in patients diagnosed with schizophrenia in comparison with general population [37,39-42], although in one study no such relationship was found [43].

Nucleotide sequences homologous to those of known retroviruses were detected in the CSFs of individuals diagnosed with acute or chronic schizophrenia [39]. Moreover, particles that contain HERV-W RNA have been identified in the plasma of patients with recent onset of the disorder [40]. However, to date it is still not known whether these results are the consequence of qualitative or quantitative differences in the transcription of genomic regions that harbor HERV-W elements.

Along similar lines, elevated levels of gag transcripts have been described in peripheral blood mononuclear cells from patients after a first episode of schizophrenia. It should be mentioned that this cell population could be useful to identify markers of the disease [44] due to the changes in gene expression described in these cells in patients diagnosed with schizophrenia. Likewise, in correlation with clinical aspects of the disease, elevated levels of transcripts from the HERV-W gag found in the 11q13.5 chromosome region were only evident in those individuals who are suffering the transition from susceptibility to manifestation of symptoms [37].

By contrast and in relation with the translation of HERV-W transcripts, gag protein expression was reduced in post mortem tissue from several human brain regions when compared with control samples [42]. Positive Antigens for env and gag have been described in the serum of schizophrenic patients, confirming the previous 
Table 1. Summary of the principal findings of the relationships between schizophrenia and HERV-W.

\begin{tabular}{|c|c|c|c|c|}
\hline Year & Author(s) & $\begin{array}{c}\text { Sample size } \\
\text { (patients/controls) }\end{array}$ & Material studied & Findings (compared to controls) \\
\hline 2001 & Karlsson et al. & $55 / 52^{1}$ & Cerebrospinal fluids & $\begin{array}{l}\text { Identified pol transcripts of some HERV families in } \\
\text { individuals with acute onset of schizophrenia and } \\
\text { individuals with chronic schizophrenia. }\end{array}$ \\
\hline 2004 & Karlsson et al. & $54 / 46$ & Plasma & $\begin{array}{l}\text { HERV-W related RNA in the plasma of } 16.6 \% \text { of recently } \\
\text { hospitalized patients diagnosed of schizophrenia. }\end{array}$ \\
\hline 2005 & Frank et al. & 77/39 & Brain samples & $\begin{array}{l}\text { Characteristic brain-specific retroviral activity profile } \\
\text { (members of the families HERV-E, HERV-F, ERV9 and } \\
\text { HERV-K). }\end{array}$ \\
\hline 2007 & Weis et al. & $15 / 15$ & Brain samples & $\begin{array}{l}\text { Reduced expression of HERV-W gag protein in } \\
\text { schizophrenic patients }\end{array}$ \\
\hline 2008 & Yao et al. & $30 / 26$ & Peripheral blood mononuclear cells & $\begin{array}{l}\text { Elevated levels of HERV-W gag transcripts in peripheral } \\
\text { blood mononuclear cells from patients with a first episode } \\
\text { of schizophrenia. }\end{array}$ \\
\hline 2008 & Perron et al. & $49 / 49$ & Serum & $\begin{array}{l}\text { Presence of HERV-W proteins in the serum of } \\
\text { schizophrenic patients. }\end{array}$ \\
\hline
\end{tabular}

Table 2. Summary of the principal findings of the relationships between schizophrenia and HERV-K.

\begin{tabular}{llcll}
\hline Year & Author(s) & $\begin{array}{c}\text { Sample size } \\
\text { (patients/controls) }\end{array}$ & Material studied & Findings (compared to controls) \\
\hline 2005 & Frank et al. & $77 / 39$ & Brain samples & $\begin{array}{l}\text { Characteristic brain-specific retroviral activity profile } \\
\text { (members of the families HERV-E, HERV-F, ERV9 and } \\
\text { HERV-K). } \\
\text { HERV-K115 not associated with schizophrenia in } \\
\text { Japanese population, but the frequency of this HERV } \\
\text { might be increased in patients with younger onset. }\end{array}$ \\
\hline Otowa et al. & 178/181 & DNA samples & $\begin{array}{l}\text { Strong association between the risk of type 2 diabetes in } \\
\text { schizophrenic patients and genetic polymorphisms within } \\
\text { the envelope region of the HERV K-18. }\end{array}$ \\
\hline
\end{tabular}

reports of specific virion-associated HERV-W RNA in CSF and at the protein level in plasma, as mentioned above [41].

Finally, the env gene of HERV-W is thought to be transcriptionally active, even though there is no significant difference in env transcription between healthy controls and schizophrenic patient samples [43].

\subsection{HERV-K and Schizophrenia}

The HERV-K family is that which most recently integrated into the human genome, occurring approximately 5 million years ago. It has been associated with different human diseases, as it is comprised of biologically active retroelements that have undergone polymorphic integration events in the human population [45]. To date, it is the only know retroviral family with open reading frames encoding the different HERV proteins and that is capable of producing intact viral particles [46]. HERV-K expression has been linked to different types of cancer and like HERV-W, it has been associated with the non-pathological human placenta [24]. In addition, it has been found in normal human brain, where it was identified at the chromosome region 1q21-q22, retaining promoter/ enhancer activity.

Although the role of HERV-K in the pathogenesis of schizophrenia has not been studied as extensively as that of HERV-W, there is evidence from post mortem material and DNA samples that the HERV-K family may be implicated in this disease (Table 2) $[44,47,48]$. Indeed, the potential association of HERV-K with schizophrenia [43] was evident through the overrepresentation of pol transcripts from this HERV in schizophrenic patients when compared with unaffected individuals.

\section{FUTURE DIRECTIONS}

We believe that in the following years, studying the biology of HERVs will be at the forefront of molecular neurobiological research into schizophrenia. As indicated above, there is important data that points to the involvement of HERVs in the pathogenesis of schizophrenia, mainly with regards to HERV-W.

Much progress has been made in the diagnosis, classi- 
fication and treatment of schizophrenia. However, since the promoters of endogenous retroviruses contain binding sites for a variety of transcription factors [49], we believe that their study, and that of the epigenetic status of those HERVs related to the disease, could provide important data regarding the molecular etiology of this disease, a phenomenon that is still not completely understood $[34,50]$.

Along these lines, mRNA expression of HERVs has been seen to be stimulated by steroid hormone treatment, providing evidence that enhanced expression of the HERV genome by steroid hormones may be involved in the etiology of a human disease [19]. With regards schizophrenia, and focusing on the gender and age characteristics of the disorder, the peak incidence for males and females is between $15-24$ years of age, females also showing a second peak incidence at age 55 - 64 [51]. Bearing in mind that both periods coincide with moments of significant hormonal changes, it has been proposed that these changes may produce an enhanced expression of the HERV families implicated in the etiopathology of schizophrenia [34]. Thus, it will be necessary to generate sufficient experimental data to help us better understand the molecular etiology of schizophrenia. In this respect, and as we said before transcriptome profiling using deep-sequencing approaches such as RNA-Seq $[52,53]$ will be essential, alongside post mortem, genetic linkage and mutation analysis, as well as in vitro experimental studies.

\section{REFERENCES}

[1] Baltimore, D. (1970). Rna-dependent DNA polymerase in virions of RNA tumour viruses. Nature, 226, 1209-1211. doi:10.1038/2261209a0

[2] Temin, H.M. and Mizutani, S. (1970) Rna-dependent DNA polymerase in virions of rous sarcoma virus. Nature, 226, 1211-123. doi:10.1038/2261211a0

[3] Martin, M.A., Bryan, T., Rasheed, S. and Kahn, A.S. (1981) Identification and cloning of endogenous retroviral sequences present in human DNA. Proceedings of the National Academy of Sciences of the United States of America, 78, 4892-4896. doi:10.1073/pnas.78.8.4892

[4] Bonner, T.I, O’Connell, C. and Cohen, M. (1982) Cloned endogenous retroviral sequences from human DNA. Proceedings of the National Academy of Sciences of the United States of America, 79, 4709-4713.

doi:10.1073/pnas.79.15.4709

[5] Lander, E.S., Linton, L.M., Birren, B., et al. (2001) international human genome sequencing consortium. Initial sequencing and analysis of the human genome. Nature, 409, 860-921. doi:10.1038/35057062

[6] Venter, J.C., Adams, M.D., Myers, E.W., et al. (2001) The sequence of the human genome. Science, 291, 1304-1351. doi:10.1126/science. 1058040
[7] Lower, R., Lower, J. and Kurth, R. (1996) The viruses in all of us: Characteristics and biological significance of human endogenous retrovirus sequences. Proceedings of the National Academy of Sciences of the United States of America, 93, 5177-5184. doi:10.1073/pnas.93.11.5177

[8] Baumann, J.G. (2006) Intracellular restriction factors in mammalian cells-An ancient defense system finds a modern foe. Current HIV Research, 4, 141-168. doi:10.2174/157016206776055093

[9] Towers, G.J. (2005) Control of viral infectivity by tripartite motif proteins. Human Gene Therapy, 16, 1125-1132. doi:10.1089/hum.2005.16.1125

[10] Cullen, B.R. (2006) Role and mechanism of action of the apobec3 family of antiretroviral resistance factors. Journal of Virology, 80, 1067-1076. doi:10.1128/JVI.80.3.1067-1076.2006

[11] Blikstad, V., Benachenhou, F., Sperber, G.O. and Blomberg, J. (2008) Evolution of human endogenous retroviral sequences: A conceptual account. Cellular and Molecular Life Sciences, 65, 3348-3365. doi:10.1007/s00018-008-8495-2

[12] Eiden, M.V. (2008) Endogenous retroviruses-aiding and abetting genomic plasticity. Cellular and Molecular Life Sciences, 65, 3325-3328. doi:10.1007/s00018-008-8493-4

[13] Rebora, A. (2005) Human endogenous retroviruses and their possible impact on dermatology. Journal of the American Academy of Dermatology, 52, E7. doi:10.1016/S0190-9622(03)02540-4

[14] Sverdlov, E.D. (2000) Retroviruses and primate evolution. Bioessays, 22, 161-171. doi:10.1002/(SICI)1521-1878(200002)22:2<161::AID-BI ES7>3.0.CO;2-X

[15] Flockerzi, A., Ruggieri, A., Frank, O., Sauter, M., Maldener, E., Kopper, B., Wullich, B., Seifarth, W., MüllerLantzsch N., Leib-Mösch C., Meese E. and Mayer J. (2008) Expression patterns of transcribed human endogenous retrovirus herv-k (hml-2) loci in human tissues and the need for a herv transcriptome project. $B M C \mathrm{Ge}$ nomics, 9, 354. doi:10.1186/1471-2164-9-354

[16] Khodosevich, K., Lebedev, Y. and Sverdlov, E. (2002) Endogenous retroviruses and human evolution. Comparative and Functional Genomics, 3, 494-498. doi:10.1002/cfg.216

[17] Hohenadl, C., Germaier, H., Walchner, M., Hagenhofer, M., Herrmann, M., Stürzl, M., Kind, P., Hehlmann, R., Erfle, V. and Leib-Mösch, C. (1999) Transcriptional activation of endogenous retroviral sequences in human epidermal keratinocytes by UVB irradiation. Journal of Investigative Dermatology, 113, 587-594. doi:10.1046/j.1523-1747.1999.00728.x

[18] Nellaker, C., Yao, Y., Jones-Brando, L., Mallet, F., Yolken, R.H. and Karlsson, H. (2006) Transactivation of elements in the human endogenous retrovirus $\mathrm{w}$ family by viral infection. Retrovirology, 3, 44. doi:10.1186/1742-4690-3-44

[19] Ono, M., Kawakami, M. and Ushikubo, H. (1987) Stimulation of expression of the human endogenous retrovirus genome by female steroid hormones in human breast 
cancer cell line t47d. Journal of Virology, 61, 2059-2062.

[20] Nelson, P.N., Hooley, P., Roden, D., Davari, E., Rylance, P., Warren, P., Martin, J. and Murray, P. G. (2004) Human endogenous retroviruses: Transposable elements with potential? Clinical and Experimental Immunology, 138, 1-9. doi:10.1111/j.1365-2249.2004.02592.x

[21] Taruscio, D. and Mantovani, A. (1998) Human endogenous retroviruses and environmental endocrine disrupters: A connection worth exploring? Teratology, 58, 27-28. doi:10.1002/(SICI)1096-9926(199808)58:2<27::AID-TE $\underline{\text { RA2>3.0.CO;2-Y }}$

[22] Schon, U., Seifarth, W., Baust, C., Hohenadl, C., Erfle, V. and Leib-Mösch, C. (2001) Cell type-specific expression and promoter activity of human endogenous retroviral long terminal repeats. Virology, 279, 280-291. doi:10.1006/viro.2000.0712

[23] Majors, J. (1990) The structure and function of retroviral long terminal repeats. Current Topics in Microbiology and Immunology, 157, 49-92. doi:10.1007/978-3-642-75218-6_3

[24] Mi, S., Lee, X., Li, X., Veldman, G.M., Finnerty, H., Racie, L., LaVallie, E., Tang, XY., Edouard, P., Howes, S., Keith, J. C. Jr. and McCoy, J. M. (2000) Syncytin is a captive retroviral envelope protein involved in human placental morphogenesis. Nature, 403, 785-789. doi:10.1038/35001608

[25] Lower, R. (1999) The pathogenic potential of endogenous retroviruses: Facts and fantasies. Trends in Microbiology, 7, 350-356. doi:10.1016/S0966-842X(99)01565-6

[26] Dewannieux, M., Harper, F., Richaud, A., Letzelter, C., Ribet, D., Pierron, G. and Heidmann, T. (2006) Identification of an infectious progenitor for the multiple-copy herv-k human endogenous retroelements. Genome Research, 16, 1548-1556. doi:10.1101/gr.5565706

[27] Kazazian, H.H. Jr. (1998) Mobile elements and disease. Current Opinion in Genetics \& Development, 8, 343-350. doi:10.1016/S0959-437X(98)80092-0

[28] Colmegna, I. and Garry, R. F. (2006) Role of endogenous retroviruses in autoimmune diseases. Infectious Disease Clinics of North America, 20, 913-929. doi:10.1016/j.idc.2006.09.008

[29] Mullins, C.S. and Linnebacher, M. (2011) Endogenous retrovirus sequences as a novel class of tumor-specific antigens: An example of HERV-H env encoding strong CTL epitopes. Cancer Immunology Immunotherapy, ahead of print. doi:10.1007/s00262-011-1183-3

[30] Buzdin, A. (2007) Human-specific endogenous retroviruses. Scientific World Journal, 7, 1848-1868. doi:10.1100/tsw.2007.270

[31] An, D. S., Xie, Y. and Chen, I. S. (2001) Envelope gene of the human endogenous retrovirus herv-w encodes a functional retrovirus envelope. Journal of Virology, 75, 3488-3489. doi:10.1128/JVI.75.7.3488-3489.2001

[32] Balada, E., Vilardell-Tarrés, M. and Ordi-Ros, J. (2010) Implications of human endogenous retroviruses in the development of autoimmune diseases. International Reviews of Immunology, 29, 351-370. doi:10.3109/08830185.2010.485333
[33] Lewis, D.A. (2001) Retroviruses and the pathogenesis of schizophrenia. Proceedings of the National Academy of Sciences of the United States of America, 98, 4293-4294. doi:10.1073/pnas.081075898

[34] Iturrieta-Zuazo, I. and Alelú-Paz, R. (2009) Human endogenous retroviruses, hormones and apobec3g: A connection to explore in schizophrenia. Bioscience Hypotheses, 2, 236-239. doi:10.1016/j.bihy.2009.04.006

[35] Huang, W. J., Liu, Z. C., Wei, W., Wang, G. H., Wu, J. G. and Zhu, F. (2006) Human endogenous retroviral pol rna and protein detected and identified in the blood of individuals with schizophrenia. Schizophrenia Research, 83, 193-199. doi:10.1016/j.schres.2006.01.007

[36] Katzourakis, A. and Tristem, M. (2005) Phylogeny of human endogenous and exogenous retroviruses. In: Sverdlov, E. Ed., Retroviruses and Primate Genome Evolution, Landes Bioscience, Georgetown, 186-203.

[37] Yao, Y., Schroder, J., Nellaker, C., Bottmer, C., Bachmann, S., Yolken, R. H. and Karlsson, H. (2008) Elevated levels of human endogenous retrovirus-w transcripts in blood cells from patients with first episode schizophrenia. Genes, Brain and Behavior, 7, 103-112.

[38] Kim, H.S., Ahn, K. and Kim, D.S. (2008) Quantitative expression of the herv-w env gene in human tissues. $A r$ chives of Virology, 153, 1587-1591. doi:10.1007/s00705-008-0159-X

[39] Karlsson, H., Bachmann, S., Schroder, J., McArthur, J., Torrey, E.F. and Yolken R.H. (2001) Retroviral ma identified in the cerebrospinal fluids and brains of individuals with schizophrenia. Proceedings of the National Academy of Sciences of the United States of America, 98, 46344639. doi:10.1073/pnas.061021998

[40] Karlsson, H., Schroder, J., Bachmann, S., Bottmer, C., and Yolken, R.H. (2004) Herv-w-related rna detected in plasma from individuals with recent-onset schizophrenia or schizoaffective disorder. Molecular Psychiatry, 9, 1213. doi:10.1038/sj.mp.4001439

[41] Perron, H., Mekaoui, L., Bernard, C., Veas, F., Stefas, I. and Leboyer, M. (2008) Endogenous retrovirus type w gag and envelope protein antigenemia in serum of schizophrenic patients. Biological Psychiatry, 64, 1019-1023. doi:10.1016/j.biopsych.2008.06.028

[42] Weis, S., Llenos, I.C., Sabunciyan, S., Dulay, J. R., Isler, L., Yolken, R. and Perron, H. (2007) Reduced expression of human endogenous retrovirus (herv)-w gag protein in the cingulate gyrus and hippocampus in schizophrenia, bipolar disorder, and depression. Journal of Neural Transmission, 114, 645-655. doi:10.1007/s00702-006-0599-y

[43] Frank, O., Giehl M., Zheng C., Helhmann R., LeibMösch C. and Seifarth W. (2005) Human endogenous retrovirus expression profiles in samples from brains of patients with schizophrenia and bipolar disorders. Journal of Virology, 79, 10890-10901. doi:10.1128/JVI.79.17.10890-10901.2005

[44] Bowden, N.A., Weidenhofer, J., Scott, R.J., Schall, U., Todd, J., Michie, P.T. and Tooney, P.A. (2006) Preliminary investigation of gene expression profiles in peripheral blood lymphocytes in schizophrenia. Schizophrenia Research, 82, 175-183. doi:10.1016/j.schres.2005.11.012 
[45] Serafino, A., Balestrieri, E., Pierimarchi, P., Matteucci, C., Moroni, G., Oricchio, E., Rasi, G., Mastino, A., Spadafora, C., Garaci, E. and Vallebona, P.S. (2009) The activation of human endogenous retrovirus $\mathrm{k}$ (herv-k) is implicated in melanoma cell malignant transformation. Experimental Cell Research, 315, 849-862. doi:10.1016/j.yexcr.2008.12.023

[46] Boller, K., Schonfeld, K., Lischer, S., Fischer, N., Hoffmann, A., Kurth, R. and Tönjes, R.R. (2008) Human endogenous retrovirus herv-k113 is capable of producing intact viral particles. Journal of General Virology, 89, 567572. doi:10.1099/vir.0.83534-0

[47] Dickerson, F., Rubalcaba, E., Viscidi, R., Yang, S., Stallings, C., Sullens, A., Origoni, A., Leister, F. and Yolken, R. (2008) Polymorphisms in human endogenous retrovirus k-18 and risk of type 2 diabetes in individuals with schizophrenia. Schizophrenia Research, 104, 121-126. doi:10.1016/j.schres.2008.05.005

[48] Otowa, T., Tochigi, M., Rogers, M., Umekage, T., Kato, N. and Sasaki T. (2006) Insertional polymorphism of en- dogenous retrovirus herv-k115 in schizophrenia. Neuroscience Letters, 408, 226-229.

doi:10.1016/j.neulet.2006.09.004

[49] Portis, J.L. (2002) Perspectives on the role of endogenous human retroviruses in autoimmune diseases. Virology, 296, 1-5. doi:10.1006/viro.2002.1388

[50] Leung, D.C. and Lorincz, M.C. (2011) Silencing of endogenous retroviruses: When and why do histone marks predominate? Trends in Biochemical Sciences, in press. doi:10.1016/j.tibs.2011.11.006

[51] Munk-Jorgensen, P. (1987) First-admission rates and marital status of schizophrenics. Acta Psychiatrica Scandinavica, 76, 210-216. doi:10.1111/j.1600-0447.1987.tb02886.x

[52] Wang, Z. (2009) RNA-Seq: A revolutionary tool for transcriptomics. Nature Reviews Genetics, 10, 57-63. doi:10.1038/nrg2484

[53] Wang, Z. (2009) RNA-Seq: Advances, challenges and opportunities. Nature Reviews Genetics, 12, 87-98. 